\title{
Antiepileptic and anti-neuroinflammatory effects of red ginseng in an intrahippocampal kainic acid model of temporal lobe epilepsy demonstrated by electroencephalography
}

\author{
Ju Young Kim¹, Jin Hyeon Kim¹, Hee Jin Lee ${ }^{1}$, Sang Hoon Kim', Young Jin Jung ${ }^{2}$, \\ Hee-Young Lee ${ }^{3}$, Hee Jaung $\mathrm{Kim}^{3}$, Sae Yoon $\mathrm{Kim}^{1}$ \\ Departments of ${ }^{1}$ Pediatrics and ${ }^{2}$ Neurosurgery, Yeungnam University College of Medicine; \\ ${ }^{3}$ Laboratory Animal Center, Daegu-Gyeongbuk Medical Innovation Foundation, Daegu, Korea
}

Background: Chronic inflammation can lower the seizure threshold and have influence on epileptogenesis. The components of red ginseng (RG) have anti-inflammatory effects. The abundance of peripherally derived immune cells in resected epileptic tissue suggests that the immune system is a potential target for anti-epileptogenic therapies. The present study used continuous electroencephalography (EEG) to evaluate the therapeutic efficacy of RG in intrahippocampal kainic acid (IHKA) animal model of temporal lobe epilepsy.

Methods: Prolonged status epilepticus (SE) was induced in 7-week-old C57BL/6J mice via stereotaxic injection of kainic acid (KA, $150 \mathrm{~nL} ; 1 \mathrm{mg} / \mathrm{mL})$ into the right $\mathrm{CA} 3 /$ dorsal hippocampus. The animals were implanted electrodes and monitored for spontaneous seizures. Following the IHKA injections, one group received treatments of RG (250 mg/kg/day) for 4 weeks (RG group, $n=7)$ while another group received valproic acid (VPA, $30 \mathrm{mg} / \mathrm{kg} /$ day) (VPA group, $\mathrm{n}=7$ ). Laboratory findings and pathological results were assessed at D29 and continuous (24 h/week) EEG monitoring was used to evaluate high-voltage sharp waves on D7, D14, D21, and D28.

Results: At D29, there were no differences between the groups in liver function test but RG group had higher blood urea nitrogen levels. Immunohistochemistry analyses revealed that RG reduced the infiltration of immune cells into the brain and EEG analyses showed that it had anticonvulsant effects.

Conclusion: Repeated treatments with RG after IHKA-induced SE decreased immune cell infiltration into the brain and resulted in a marked decrease in electrographic seizures. RG had anticonvulsant effects that were similar to those of VPA without serious side effects.

Keywords: Electroencephalography; High voltage sharp wave; Intrahippocampal kainic acid model; Red ginseng; Temporal lobe epilepsy

Received: October 22, 2018, Revised: Octover 31, 2018 Accepted: October 31, 2018

Corresponding Author: Sae Yoon Kim, Department of Pediatrics, Yeungnam University College of Medicine, 170, Hyeonchung-ro, Nam-gu, Daegu 42415, Korea Tel: +82-53-620-3533, Fax: +82-53-629-2252

E-mail: sysnow88@hanmail.net

\section{INTRODUCTION}

Epilepsy is a chronic neurological condition characterized by recurrent, unprovoked seizures [1]. According to the World Health Organization, epilepsy is the most prevalent neurological disorder worldwide, affecting over 50 million people, with 2.4 million new cases annually [2].

Copyright $($ C) 2018 Yeungnam University College of Medicine

This is an Open Access article distributed under the terms of the Creative Commons Attribution Non-Commercial License (http://creativecommons.org/licenses/by-nc/4.0/) which permits unrestricted non-commercial use, distribution, and reproduction in any medium, provided the original work is properly cited. 
Many antiepileptic drugs (AEDs) are available to control or reduce seizures, but approximately one third of patients are refractory to medication [3]. Surgical resection is one treatment option, but the seizure zone and possible post-surgical neurological deficits must be assessed with multiple expensive tests, including invasive pre-surgical procedures such as intracranial electroencephalography (EEG) [2].

Kainic acid $(\mathrm{KA})$ is a potent glutamate analog used to induce neurodegeneration in rodents [1]. Intracerebral injections of $\mathrm{KA}$ in the hippocampus or amygdala produce temporal lobe epilepsy (TLE) that emulates the histopathological changes seen in epileptic patients [2].

Red ginseng (RG) (Panax ginseng; harvested after at least 6 years) has anti-inflammatory effects and fewer side effects than fresh or white ginseng [4]. Korean red ginseng (KRG) is an effective natural drug that possesses various biological activities, including boosting the immune system [1,5,6]. Ginsenosides are the main ingredients of KRG and their value in neurodegenerative disease has been studied extensively [7].

We examined the anticonvulsant effects of KRG with EEG in an animal model of refractory epilepsy.

\section{MATERIAL AND METHODS}

\section{Animal}

Young male C57BL/6J mice (7-week-old, 18-20 g) were obtained from the Animal Center of Daegu-Gyeongbuk Medical Innovation Foundation and housed at a constant temperature of $23^{\circ} \mathrm{C}$ with a 12 hours light-dark cycle (light on from 07:00 to 19:00). All animals were provided with ad libitum food and water. We performed 16 intrahippocampal kainic acid (IHKA) trials and divided them into two groups according to their weight and two of them were observed without any treatment. Three of them were dead with status epilepticus (SE) on D1 and excluded on the next day. We had six animals for each group and one more for observation.

\section{Animal treatment}

The mice received daily oral administrations of either KRG extract $(250 \mathrm{mg} / \mathrm{kg}, \mathrm{n}=6)$ or valproic acid (30 mg/kg, $\mathrm{n}=6$ ) for 28 days and then brain and serum samples were collected 1 day after the final administration. In this model, SE was induced by a unilateral injection of KA (1:1000; $150 \mathrm{~nL}$, SigmaAldrich; Steinheim, Germany) stereotaxically administered to the right CA3 area (AP: -1.7, ML: -1.7, and DB: -1.8 ) of the dorsal hippocampus after anesthesia was induced using chloral hydrate. The injections were slowly administered over $120 \mathrm{~s}$ using a microsyringe and a nanoinjector controller and the microsyringe was left in place for an additional $120 \mathrm{~s}$ after the injection to prevent backflow.

Next, a screwdriver was used to place the recording electrodes in the burr hole in such a way that the tips of the electrodes contacted the brain surface. The electrodes were attached to the pin connecter, the electrode wire was gently wound up, and then the wire was connected to the EEG amplifiers.

\section{EEG monitoring}

The EEG data were continuously monitored $24 \mathrm{~h} /$ week using the MP160 \& EEG 100C systems (BIOPAC Systems). The EEG data were visually examined to identify abnormal electrographic activity and the occurrence of epileptiform discharges were assessed to quantify the development of epileptogenesis.

\section{Laboratory finding}

Blood samples were obtained at D29 and then subjected to a centrifugal separator. Subsequently, serum samples were analyzed with the AU 5400 chemistry analyzer (Olympus Optical Co. Ltd; Tokyo, Japan) to measure aspartate aminotransferase (AST), alanine aminotransferase (ALT), blood urea nitrogen (BUN), and creatinine (Cre) levels to determine side effects; serum levels were averaged for comparisons.

\section{Histological evaluation and immunohistochemistry}

The tissues of both groups were stored in a formaldehyde solution and fixed with paraffin. Subsequently, hematoxylin and eosin (H\&E) staining was performed and immunohistochemical analyses were conducted by incubating the tissues with rabbit anti-glial fibrillary acidic protein (GFAP; 1:5000, Dako; Carpinteria, CA, USA) 


\section{Ethical statement}

All experimental procedures were reviewed and approved by the Institutional Animal Care and Use Committee of Daegu-Gyeongbuk Medical Innovation Foundation (DGMIF18102301).

\section{Statistical analysis}

All statistical analyses were performed using IBM SPSS version 21.0 (IBM Co., Armonk, NY, USA). Data were evaluated with Student $\mathrm{t}$-tests and repeated-measures analysis of variance (ANOVA) tests. All data are presented as mean \pm standard deviation and $p$-values $<0.05$ were considered to indicate statistical significance.

\section{RESULTS}

The present study performed 16 IHKA trials that were categorized into two groups according to weight; two animals were observed without any treatment and three died and were excluded from the experiments the next day.

\section{Laboratory findings}

Laboratory findings and pathological results were assessed at D29. There were no significant differences in serum levels

Table 1 . The laboratory findings of each group

\begin{tabular}{lllc}
\hline Variable & VPA $(\mathrm{n}=6)$ & $\mathrm{RG}(\mathrm{n}=6)$ & $p$-value \\
\hline AST $(\mathrm{U} / \mathrm{L})$ & $63.5 \pm 11.2$ & $70.0 \pm 17.8$ & 0.431 \\
ALT $(\mathrm{U} / \mathrm{L})$ & 24.6 & 18.8 & 0.222 \\
BUN $(\mathrm{mg} / \mathrm{dL})$ & $20.2 \pm 2.7$ & $16.2 \pm 2.6$ & 0.022 \\
Cre $(\mathrm{mg} / \mathrm{dL})$ & $0.1 \pm 0.0$ & $0.0 \pm 0.0$ & 0.250 \\
\hline
\end{tabular}

VPA, valploic acid; RG, red ginseng; AST, aspartate aminotransferase, ALT, alanine aminotransferase; BUN, blood urea nitrogen; Cre, creatine. of AST or ALT between the VPA and RG groups (63.5 U/L vs. $70.0 \mathrm{U} / \mathrm{L}$, respectively). However, BUN levels were lower in the RG group $(16.2 \mathrm{mg} / \mathrm{dL})$ than in the VPA group (20.2 $\mathrm{mg} / \mathrm{dL}, p=0.022$ ). There were no significant differences in Cre levels between the two groups (Table 1).

\section{Histology}

Histopathological assessments of lesions in animals in the IHKA model were made and compared between the two groups. The H\&E results revealed no definite neuronal cell loss in either of the groups (Figs. 1A, 1B), but the GFAP analyses revealed that there were more astrocytes which were showed with neuronal damages in the VPA group than RG group (Figs. 1C, 1D).

\section{Electroencephalography}

Continuous (24 h/week) EEG monitoring was employed to assess high-voltage sharp waves (HVSWs) on D7, D14, D21, and D28, and the number of epileptiform discharges were counted as HVSWs (Fig. 2). A novel definition of HVSWs was used: sharp waves with a high amplitude at least three times the EEG baseline, a duration of at least $5 \mathrm{~s}$, and a frequency of at least $2 \mathrm{~Hz}$ [8]. The control animal recorded an average of HVSWs per hour 16.0 times over 4 weeks, and the results are 17, 21, 12, and 14 weekly. RG group showed $17.1 \pm 9.2,13.0 \pm 7.9,6.9 \pm 5.2$, and $4.4 \pm 3.7$ each week and VPA group showed 13.5 $\pm 12.0,16.6 \pm 12.6,14.3 \pm 13.9$, and $9.5 \pm 10.5$ each week. These records showed that anticonvulsant effects were effective over time in both groups (Table 2, $p<0.001)$. The EEG data revealed that the anticonvulsant effects of RG were similar to those of VPA (Fig. 3, $p=0.466$ ).

\section{DISCUSSION}

Many AEDs are currently available to control or reduce seizures, but approximately one third of patients are refrac-

Table 2. The comparison between groups with the frequency of HVSWs per hour

\begin{tabular}{cccccc}
\hline Group & 1 st wk & 2nd wk & 3rd wk & 4th wk & $p$-value \\
\hline VPA $(n=6)$ & $13.5 \pm 12.0$ & $16.6 . \pm 12.6$ & $14.3 \pm 13.9$ & $9.5 \pm 10.5$ & $<0.001$ \\
RG $(n=6)$ & $17.1 \pm 9.2$ & $13.0 \pm 7.9$ & $6.9 \pm 5.2$ & $4.4 \pm 3.7$ & \\
\hline
\end{tabular}

Values are presented as mean \pm standard deviation.

HVSW, high-voltage sharp wave; VPA, valploic acid; RG, red ginseng. 
tory to medication [3]. Although numerous AEDs have been introduced in recent decades, offering advantages in terms of tolerability and drug interactions, most clinical trials and meta-analyses have failed to demonstrate any superiority of these newer AEDs over older drugs in terms of efficacy [9]. The main therapeutic modality of refractory epilepsy is rational polytherapy [10], while several surgical treatments have improved the outcomes of patients with drug-resistant epilepsy [9]. Nevertheless, refractory epilepsy is still a serious condition associated with many social challenges.

TLE is the most common type of partial or localizationrelated epilepsy [10]. It is difficult to control with drugs and is often associated with severe comorbidities that may, at least in part, be a consequence of neuronal damage, particularly in the hippocampus [11]. SE is a life-threatening neurological emergency [12]. Because human TLE is the most common type of epilepsy, animal models of this condition are used to study epileptogenesis and the neuronal changes in the brain occurring after convulsions [13].

$\mathrm{KA}$ is a cyclic analog of L-glutamate and an agonist of the ionotropic KA receptor. In the early 1950s, KA was isolated and extracted from a red alga (Digenea simplex) found in tropical and subtropical waters [2]. Compared with other commonly used epilepsy models, including systemic administration of KA, the IHKA model offers several advantages [14]. In this model, the long-term EEG patterns and histological consequences are very similar to those of human mesial TLE [15]. Here, we describe local administration of KA into the mouse hippocampus using stereotaxic injection and simultaneous recording of the EEG activity. We used the

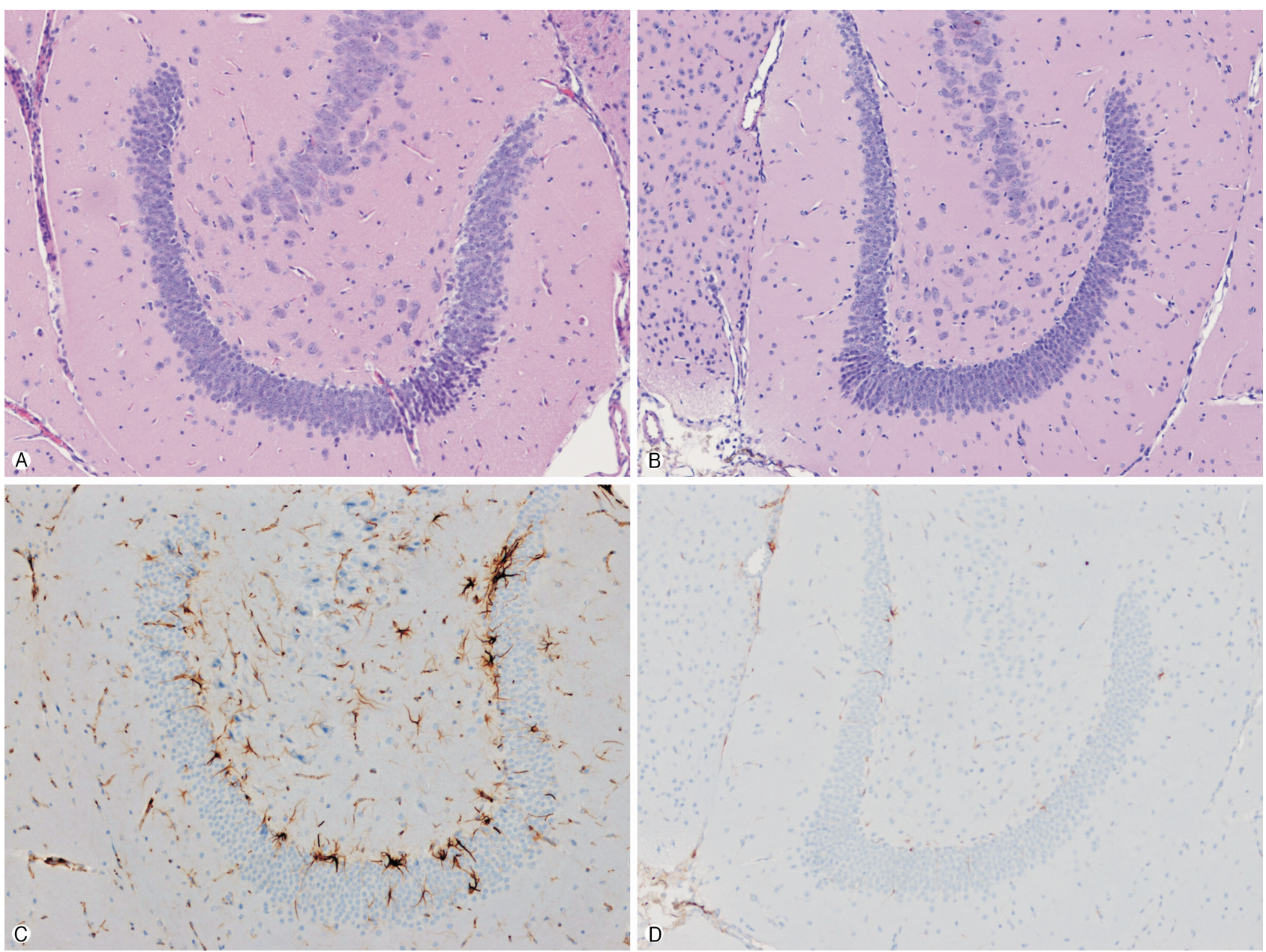

Fig. 1. Histological findings of the hippocampus after intrahippocampal kainic acid injection. There is no difference in pyramidal cell damage between valproic acid group (A) and red ginseng group (B) (hematoxylin and eosin stain, $\times 100$ ). Glial fibrillary acidic proteinpositive astrocytes are more common in valproic acid group (C) than in red ginseng group (D) (immunohistochemical stain, $\times 100$ ). 
IHKA model to identify the anticonvulsant effects in intractable epileptic animals.

The anti-inflammatory effect of RG is well known. The beneficial effects of Panax ginseng on neurodegenerative diseases have been attributed primarily to the antioxidative and immunomodulatory activities of its ginsenoside components [6. Mechanistic studies of the neuroprotective effects of ginsenosides have revealed that they act not only as antioxidants, but also as modulators of intracellular neuronal signaling and metabolism, cell survival/death genes, and mitochondrial function [6. Ginseng saponin has long served as a traditional asian medicine and is effective in treating various kinds of pain. Ginsenoside Rf is a biologically active saponin found in ginseng that has antinociceptive and anti-inflammatory effects [16].

Anticonvulsants exert their effects by acting on ion channels. There are no reports of the effects of RG on ion channels, and it is not clear whether it has anti-neuroinflammatory effects. If the anti-inflammatory effect of RG is effective at treating seizures, the efficacy of other anti-inflammatory agents or treatments may need to be tested. More research on the mechanism of these effects is needed. To confirm the anti-neuroinflammation effects of RG, GFAP staining was performed to observe astrocytes and microglial cells. Additional tests, such as Iba1 staining and measurements of cytokine blood levels, are also necessary.

In animal experiments, EEG was performed to confirm the anticonvulsant effects and the results were confirmed by video-EEG. According to previous studies, HVSWs are characterized by lower-frequency and higher-amplitude than hippocampal paroxysmal discharges [17]. We used the new definition of HVSWs, which are sharp waves with a peak amplitude of at least three times the EEG baseline, a duration of at least $5 \mathrm{~s}$, and a frequency of at least $2 \mathrm{~Hz}$ [8]. The reported number of HVSWs is 19 per hour, which is similar to the 10-20 times per hour seen in our study [8].

Valproic acid is an anticonvulsant that acts on the voltagegated sodium and calcium channels and exerts an effect on inhibitory neurotransmitters. However, it has various adverse effects, such as hepatotoxicity [12], and carries a teratogenic risk with a high rate of major fetal malformations [18]. In this study, an increase in BUN resulted from the change in

(A)

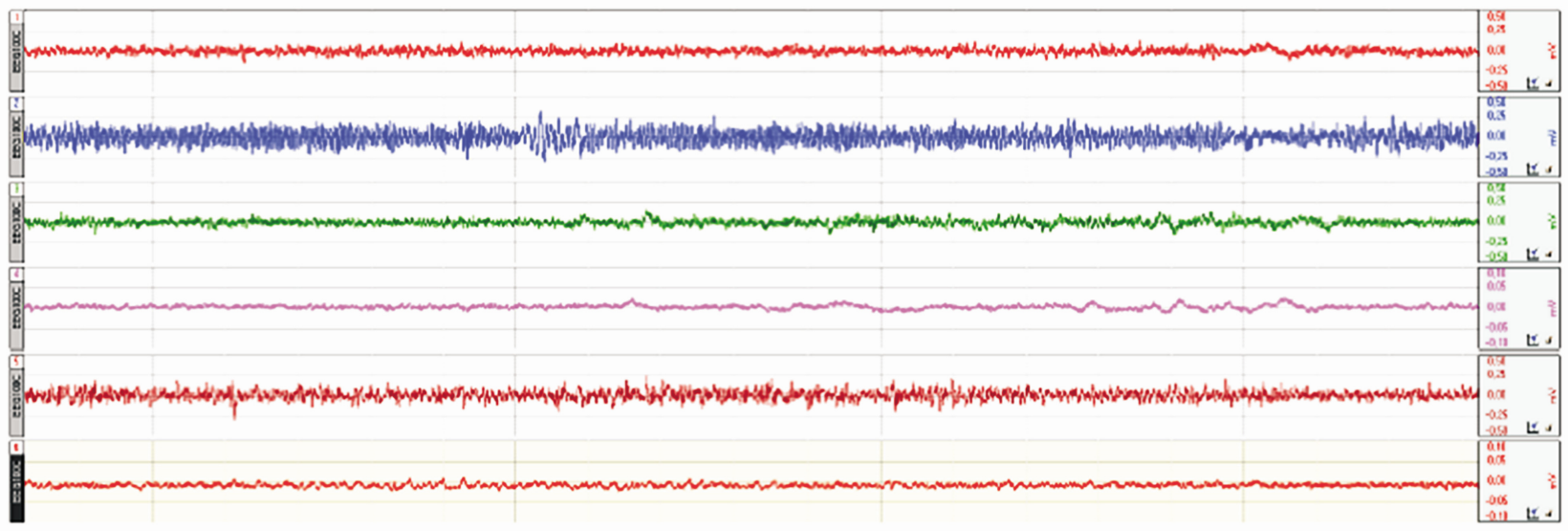

(B)

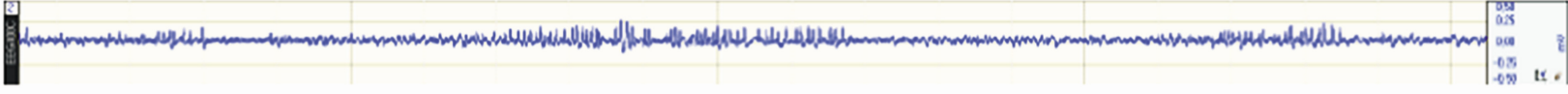

(C)
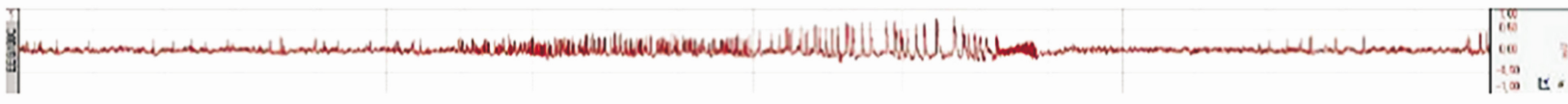

(D)
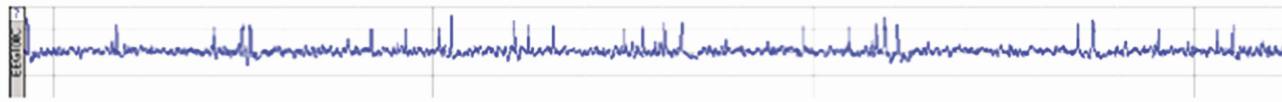

Fig. 2. Weekly continuous EEG monitorings following intrahippocampal injection of KA in male mice. The EEG recordings were performed via depth and cortical electrodes (A). Electroencephalographic findings during status epilepticus, characterized by spikes, polyspikes and spike-wave complexes. Two-thirds of the mice showed generalized seizures. (B-D) showed high-voltages sharp waves and multiple spikes in 1, 2, and 4 weeks after KA injection. EEG, electroencephalography; KA, kainic acid. 


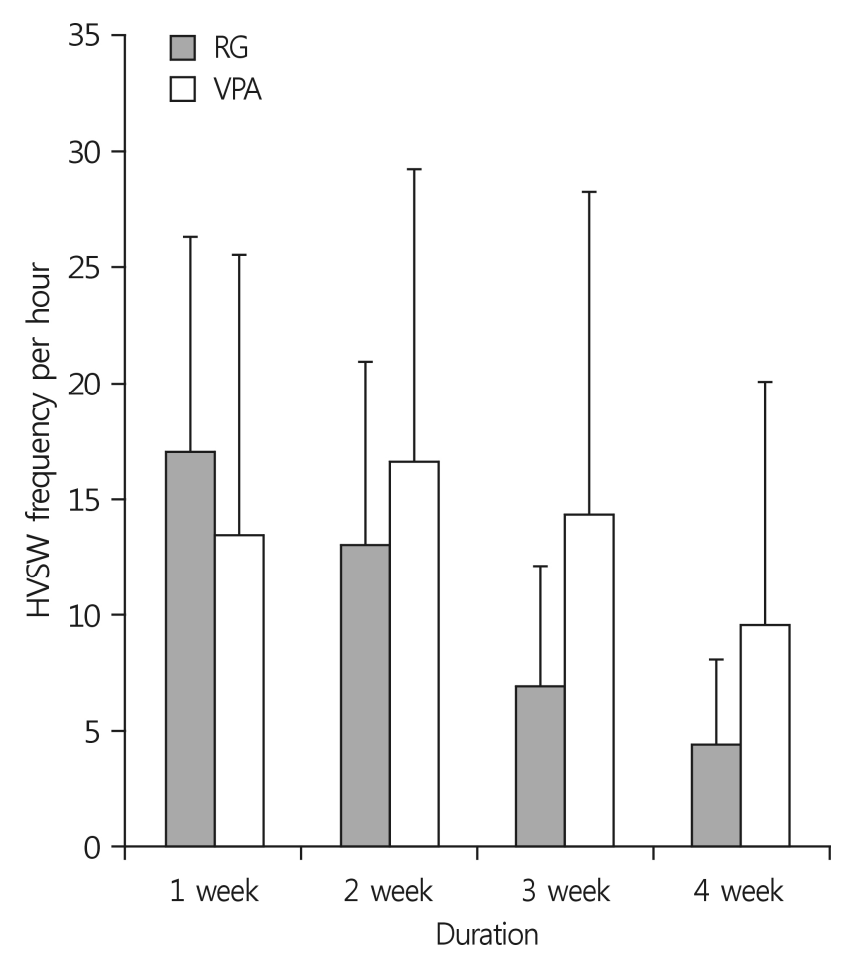

Fig. 3. Anticonvulsant effects of VPA and RG in IHKA animal model by EEG. Epileptic activity was recorded from the depth and cortical electrodes after KA injection at right CA3 region of the dorsal hippocampus. The graph shows the number of HVSWs frequency per hour weekly for 4 weeks. Overall, the number of HVSWs decreased with time, and there was no difference between the two groups ( $p=0.486$ ). VPA, valproic acid; $\mathrm{RG}$, red ginseng; IHKA, intrahippocampal kainic acid; EEG, electroencephalography; KA, kainic acid; HVSWs, high-voltages sharp waves.

oral intake of RG due to the initial SE, and was thought to indicate an acute prerenal injury caused by dehydration.

This study demonstrates that RG has anticonvulsant effects in an IHKA animal model, but it had several limitations. First, too few animals were studied to yield definitive results. Second, the tests were performed in young mice and the amounts of serum collected were small. It would be better to measure indicators of drug levels and neuroinflammation as needed. Longer-term follow-up is needed to confirm the anticonvulsant effects and other possible side effects of RG.

Repeated treatments with RG after IHKA-induced SE decreased immune cell infiltration into the brain and resulted in a marked decrease in electrographic seizures. RG had anticonvulsant effects that were similar to those of VPA and did not have any serious side effects. The present study showed anticonvulsant effects of RG in an IHKA animal model, but additional studies using other intractable epileptic animal mo- dels are necessary to further the current understanding of the mechanisms underlying these anticonvulsant effects. In addition, future studies using larger sample sizes are needed to produce clearer results.

\section{ACKNOWLEDGMENT}

This study was supported by the Dong-Won Research fund (2018-8) from Daegu Medical Association and Basic Science Research Program through the National Research Foundation of Korea (NRF) funded by the Ministry of Education (NRF 2014R1A2060062).

\section{CONFLICT OF INTEREST}

No potential conflict of interest relevant to this article was reported.

\section{ORCID}

Ju Young Kim, https://orcid.org/0000-0003-3327-9733

Jin Hyeon Kim, https://orcid.org/0000-0002-2770-1507

Hee Jin Lee, https://orcid.org/0000-0002-0687-9402

Sang Hoon Kim, https://orcid.org/0000-0002-6603-943X

Sae Yoon Kim, https://orcid.org/0000-0002-2610-0574

\section{REFERENCES}

1. Kienzler-Norwood F, Costard L, Sadangi C, Müller P, Neubert $\mathrm{V}$, Bauer S, et al. A novel animal model of acquired human te mporal lobe epilepsy based on the simultaneous administration of kainic acid and lorazepam. Epilepsia 2017;58:222-30.

2. Lévesque M, Avoli M. The kainic acid model of temporal lobe epilepsy. Neurosci Biobehav Rev 2013;37:2887-99.

3. Engel J Jr, McDermott MP, Wiebe S, Langfitt JT, Stern JM, Dewar S, et al. Early surgical therapy for drug-resistant temporal lobe epilepsy: a randomized trial. JAMA 2012;307:92230 .

4. Babiker LB, Gadkariem EA, Alashban RM, ALjohar HI. Investigation of stability of Korean ginseng in herbal drug product. Am J Appl Sci 2014;11:160-70.

5. Kim SJ, Choi S, Kim M, Park C, Kim GL, Lee SO, et al. Effect of Korean Red Ginseng extracts on drug-drug interactions. J Ginseng Res 2018;42:370-8.

6. Kim KH, Lee D, Lee HL, Kim CE, Jung K, Kang KS. Beneficial effects of Panax ginseng for the treatment and prevention 
of neurodegenerative diseases: past findings and future directions. J Ginseng Res 2018;42:239-47.

7. Choi JH, Jang M, Nah SY, Oh S, Cho IH. Multitarget effects of Korean Red Ginseng in animal model of Parkinson's disease: antiapoptosis, antioxidant, antiinflammation, and maintenance of blood-brain barrier integrity. J Ginseng Res 2018; 42:379-88.

8. Twele F, Töllner K, Brandt C, Löscher W. Significant effects of sex, strain, and anesthesia in the intrahippocampal kainate mouse model of mesial temporal lobe epilepsy. Epilepsy Behav 2016;55:47-56.

9. Conte F, Legros B, Van Paesschen W, Avbersek A, Muglia $\mathrm{P}$, Depondt C. Long-term seizure outcomes in patients with drug resistant epilepsy. Seizure 2018;62:74-8.

10. Andres-Mach M, Zagaja M, Haratym-Maj A, Rola R, Maj M, Haratym J, et al. A long-term treatment with arachidonyl-2'chloroethylamide combined with valproate increases neurogenesis in a mouse pilocarpine model of epilepsy. Int J Mol Sci 2017;18. pii: E900.

11. Thom M. Hippocampal sclerosis in epilepsy: a neuropathology review. Neuropathol Appl Neurobiol 2014;40:520-43.

12. Misra UK, Dubey D, Kalita J. Comparison of lacosamide versus sodium valproate in status epilepticus: a pilot study. Epilepsy Behav 2017;76:110-3.

13. Curia G, Lucchi C, Vinet J, Gualtieri F, Marinelli C, Torsello
A, et al. Pathophysiogenesis of mesial temporal lobe epilepsy: is prevention of damage antiepileptogenic? Curr Med Chem 2014;21:663-88.

14. Bielefeld P, Sierra A, Encinas JM, Maletic-Savatic M, Anderson A, Fitzsimons CP. A standardized protocol for stereotaxic intrahippocampal administration of kainic acid combined with electroencephalographic seizure monitoring in mice. Front Neurosci 2017;11:160.

15. Bouilleret V, Ridoux V, Depaulis A, Marescaux C, Nehlig A, Le Gal La Salle G. Recurrent seizures and hippocampal sclerosis following intrahippocampal kainate injection in adult mice: electroencephalography, histopathology and synaptic reorganization similar to mesial temporal lobe epilepsy. Neuroscience 1999;89:717-29.

16. Kim MK, Kang H, Baek CW, Jung YH, Woo YC, Choi GJ, et al. Antinociceptive and anti-inflammatory effects of ginsenoside $\mathrm{Rf}$ in a rat model of incisional pain. J Ginseng Res 2018;42:183-91.

17. Twele F, Töllner K, Brandt C, Löscher W. Significant effects of sex, strain, and anesthesia in the intrahippocampal kainate mouse model of mesial temporal lobe epilepsy. Epilepsy Behav 2016;55:47-56.

18. Lawthom C. Valproate and epilepsy: for women as well as men. Pract Neurol 2018;18:222-3. 